\title{
Traduction ou la lecture en profondeur : analyse des traductions françaises de Grande Sertão : Veredas de João Guimarães Rosa
}

\author{
Sheila Maria dos Santos \\ Universidade Federal de Santa Catarina (UFSC) \\ dossantos.sheilamaria@gmail.com
}

RÉSUMÉ: Selon André Gide, la traduction serait la manière la plus parfaite de lire un texte, aphorisme retrouvé aussi chez Muntaner, Plassard, entre autres. En effet, la lecture effectuée dans le but de servir à la traduction d'une œuvre ne possède pas les mêmes caractéristiques que la lecture pratiquée par un lecteur commun. Ainsi, en ayant conscience de l'importance de la lecture approfondie pour la traduction, ce travail se penchera sur l'analyse des deux traductions françaises de l'œuvre Grande Sertão: Veredas, de João Guimarães Rosa, intitulées Diadorim, dans le but d'identifier les différentes lectures faites du texte-source. Pour ce faire, l'on utilisera comme base théorique les œuvres de Plassard, Walter Benjamin, Ladmiral, Wuilmart, entre autres.

Mots-clés: Lecture; Traduction littéraire; Grande Sertão: Veredas.

\section{Tradução ou leitura aprofundada: análise das traduções francesas de Grande Sertão: Veredas de João Guimarães Rosa}

RESUMO: Segundo André Gide, a tradução seria a maneira mais perfeita de ler um texto, aforismo encontrado também em Muntaner, Plassard, entre outros. Com efeito, a leitura efetuada com o objetivo de servir à tradução de uma obra não possui as mesmas características que a leitura praticada pelo leitor comum. Assim, consciente da importância da leitura aprofundada para a tradução, esse trabalho partirá da análise das duas traduções francesas da obra Grande Sertão: Veredas, de João Guimarães Rosa, intituladas Diadorim, com o objetivo de identificar as diferentes leituras do texto-fonte. Para tanto, serão utilizadas como base teórica as obras de Plassard, Walter Benjamin, Ladmiral, entre outros.

Palavras-chave: leitura; tradução literária; Grande Sertão: Veredas. 


\section{Introduction}

La traduction est la forme la plus attentive de la lecture André Gide

L'intention de l'écrivain est une intention naïve, première, intuitive, celle du traducteur est une intention dérivée, dernière et de l'ordre de l'Idée

Walter Benjamin

André Gide ne fut pas le seul à croire que « la traduction est la forme la plus attentive de la lecture ", l'on retrouve aussi cet aphorisme paraphrasé chez Muntaner, lorsqu'il affirme que « la traduction est la manière la plus parfaite, la plus complète de lire », (2007, p. 21) ou encore chez Freddie Plassard, qui développe largement cette thématique dans son ouvrage Lire pour traduire (2007), dans lequel il affirme que "l'assimilation d'une traduction à une lecture va parfois jusqu'à l'idée d'une identité réversible entre lecture et traduction » (p. 20), en confirmant ainsi le lien intrinsèque entre ces deux activités qui nous concernent, au point de considérer la traduction comme une sorte de lecture approfondie, qui aurait pour but de dévoiler l'essence d'un texte en le restituant dans une autre langue.

Selon Plassard, la lecture effectuée dans le but de servir à la traduction d'une œuvre ne possède pas les mêmes caractéristiques que la lecture pratiquée par un simple lecteur, car il ne s'agit plus d'un acte visant à la compréhension personnelle, mais au déchiffrement d'un texte pour un autre public:

Lire et traduire, c'est lire pour traduire; c'est donc apprendre à déchiffrer un texte, à en cerner la spécificité grâce aux seuls éléments qui le constituent afin de pouvoir le restituer dans une autre langue, à en retenir en somme les traits pertinents à sa traduction [...] $c^{\prime}$ est insister d'abord sur la compréhension en profondeur d'un donné linguistique au moyen d'une analyse préalable. (PLASSARD, p. 8)

Selon cette perspective, où lire serait le premier contact avec la substance du texte, au travers duquel il serait possible au traducteur de restituer les particularités de l'écriture de la langue-source en produisant à son tour le textecible, l'importance de la lecture repose sur le fait qu'en tant que première étape de la traduction, elle représente pour le traducteur un acte aussi précis et pertinent qu'écrire a été pour l'écrivain. Antoine Berman affirme que «la traduction donne accès aux trésors cachés. Elle est donc révélation, manifestation de biens précieux » (2012, p. 102). Or, cette lecture qui révèle non pas à un seul 
lecteur, résultat de la lecture individuelle, mais au grand public - selon la définition de Jean-René Ladmiral (2010, p. 15) qui affirme que « la finalité d'une traduction consiste à nous dispenser de la lecture du texte original» - doit naturellement être "la forme la plus attentive », si elle souhaite "remplacer le texte-source par le "même" texte en langue-cible » (2010, p. 15).

Selon Walter Benjamin (2011) l'intention du traducteur, a contrario de celle de l'écrivain, est une intention dérivée, son regard doit creuser la parole de l'auteur à la recherche de chaque détail, aussi imperceptible qu'il soit. En ce sens, Françoise Wuilmart réaffirme la différence entre la lecture normale et la lecture réfléchie du traducteur, en rejoignant, ainsi, l'idée développée par Plassard, exposée plus haut:

Tout bon traducteur devrait commencer son travail par une analyse textuelle en profondeur. Quant à la lecture subjective, absolument courante, et attendue de tout lecteur, elle est un des sept péchés capitaux de la traduction. Toute lecture normale est une prise de sens subjective. A partir du moment où le livre quitte la table de son auteur, il ne lui appartient plus et devient protéiforme. (WUILMART, 2000, p. 47)

Or, il semble que le commentaire de Wuilmart puisse être compris sous deux primats distincts, car si d'un côté la lecture orientée vers la traduction ne doit pas s'apparenter à la lecture courante, de l'autre côté Wuilmart affirme que toute œuvre devient protéiforme une fois sortie de la table de son auteur. Ce disant, l'on conçoit la possibilité de multiples lectures, et celle du traducteur n'est pas forcément la bonne, comme l'on verra par la suite au travers de quelques extraits tirés de Grande Sertão: Veredas de João Guimarães Rosa, en langue française intitulée Diadorim. Cependant, il ne s'agit pas d'effectuer une critique négative des traductions de ces œuvres, celle condamnée par Antoine Berman, mais de se servir de quelques passages qui nous semblent pertinents pour illustrer l'importance de la lecture pour la traduction, un exemple étant souvent plus parlant qu'un long discours théorique.

\section{Lire pour traduire}

La pleine lecture, au contraire, est celle où le lecteur n'est rien de moins que celui qui veut écrire.

Roland Barthes

Plassard affirme qu'en traduction «la lecture est assimilée à la compréhension et la réécriture à la réexpression » (2007, p. 21). Or, ce n'est que 
d'un point de vue idéaliste que l'on peut dire que la lecture, la plus attentive qu'elle soit, assure la compréhension d'un texte. Il est évident que le désir de toute lecture, surtout celle qui a pour but la traduction, est de dévoiler les trésors cachés du texte et de faire tomber les barrières imposées aussi bien par les particularités de la langue-source que par celles de la langue-cible. Cependant, il existe certains cas où les retraductions d'un même texte nous laissent supposer l'existence de plusieurs textes-sources, dû à l'écart imposant entre les traductions. De même, l'on préférera assimiler la lecture à l'interprétation individuelle du texte, en rejoignant l'idée développée par Talens selon laquelle la dimension interprétative fait de la traduction « une lecture sur - et non à partir de - l'original » (apud PLASSARD, 2007, p. 20).

De ce fait, la lecture n'est jamais unique; son caractère subjectif fait d'elle une activité illimitée: 1 'on peut toujours lire autrement puisque la compréhension d'un texte reste ouverte. C'est la raison pour laquelle des écrivains tels que Rabelais, Cervantes ou Shakespeare sont encore de nos jours réinterprétés au travers de chaque retraduction de leurs œuvres, car la lecture n'épuise le texte, elle le renouvelle constamment à chaque nouveau regard qu'on lui adresse.

Pour illustrer ce propos l'on a pris un extrait du roman brésilien écrit par João Guimarães Rosa, Grande Sertão: Veredas (1956), ainsi que ses deux traductions françaises, intitulées Diadorim, la première entreprise par JeanJacques Villard (1965) et la retraduction signée Maryvonne Lapouge-Pettorelli (1996), les deux publiées chez Albin Michel. Une simple lecture des extraits nous permet d'identifier l'écart entre les textes-cibles et le texte-source :

\begin{tabular}{|c|c|c|}
\hline JGR & $\mathrm{V}, 1965$ (p. 56) & MLP, 1996 (р. 75) \\
\hline $\begin{array}{l}\text { "Sei senhor homem } \\
\text { valente, muito valente... } \\
\text { Eu precisar de homem } \\
\text { valente assim, viajar meu, } \\
\text { quinze dias, sertão } \\
\text { agora aqui muito } \\
\text { atrapalhado, gente braba, } \\
\text { tudo...". Destampei, ri } \\
\text { que ri de ouvir. }\end{array}$ & $\begin{array}{l}\text { «Je sais que vous êtes un } \\
\text { homme brave, très } \\
\text { brave... J'aurais besoin } \\
\text { d'un homme comme } \\
\text { vous, pour mon voyage, } \\
\text { pour quinze jours. Par } \\
\text { ici le sertão est pas sûr } \\
\text { en ce moment, des tas de } \\
\text { mauvaises gens, et } \\
\text { tout... ». Ah ça m'a bien } \\
\text { fait rire de l'entendre. }\end{array}$ & $\begin{array}{l}\text { «Je sais que vous êtes un } \\
\text { homme courageux, très } \\
\text { courageux... Moi avoir } \\
\text { besoin d'un homme } \\
\text { comme ça, courageux, } \\
\text { en voyage avec moi, } \\
\text { quinze jours, le sertão ici } \\
\text { maintenant très agité, } \\
\text { des gens méchants, et } \\
\text { tout... » De l'entendre, } \\
\text { j'ai pas pu me retenir, je } \\
\text { ris, mais je ris. }\end{array}$ \\
\hline
\end{tabular}

Source : l'auteure

João Guimarães Rosa structure son texte en utilisant deux registres linguistiques, mettant, ainsi, en évidence l'écart entre le langage employé par Riobaldo, un Brésilien, et Vupes, un personnage allemand. Toutefois, la première 
Traduction ou la lecture en profondeur : analyse des traductions françaises de Grande Sertão : Veredas de João Guimarães Rosa

traduction s'abstient de garder les fautes grammaticales commises par Vupes, qui caractérise pourtant son statut d'étranger au travers des erreurs de conjugaison. Le travail sur la lettre réalisé par João Guimarães Rosa, dans lequel chaque faute situe l'appartenance des personnages à un milieu distinct, disparaît au profit d'une traduction homogénéisante. De ce fait, le texte traduit perd non seulement un pourcentage de sa richesse linguistique, lorsque Jean-Jacques Villard prête le même langage à Riobaldo et à Vupes, mais aussi un peu de sa logique, car partageant le même langage, l'affirmation de Riobaldo en ce qui concerne le caractère comique $\mathrm{du}$ parler particulier de Vupes devient incohérente.

La même contradiction ne se produit pas dans la retraduction de Maryvonne Lapouge-Pettorelli, qui s'applique à conserver la distance évidente entre le langage de ces deux personnages au travers d'une traduction plus proche du texte-source comme le démontre l'extrait «Moi avoir besoin d'un homme comme ça », où la traductrice maintient l'absence de conjugaison présente dans le texte-source, «Eu precisar de homem ... », et d'autres déviations à la norme qui caractérisent le discours étranger de Vupes : «le sertão ici maintenant très agité ». Ce faisant la réaction de Riobaldo : «De l'entendre, j'ai pas pu me retenir, je ris, mais je ris », se voit ainsi justifiée de par l'écart entre la langue parlée par les Brésiliens et celle de Vupes.

Or, il est évident que les deux traducteurs ont saisi, lors de la lecture, la présence de registres linguistiques distincts dans l'extrait ci-dessus. Cependant, le lecteur de la première traduction se voit privé de cette langue sous-jacente et pourtant essentielle à la compréhension de l'extrait. Ainsi, si l'on adopte la définition de Plassard sur la fonction de la lecture, qui s'applique aussi à la traduction, lorsqu'il affirme que « lire ou traduire, c'est rétablir les liens qui font le tissu textuel, c'est retisser le texte dans toutes ses dimensions » (PLASSARD, 2007 , p. 265), l'on pourrait soutenir que la première traduction ne remplit pas la fonction accordée par le théoricien concernant la lecture et la traduction, car elle « défait le rapport sui generis que l'œuvre a institué entre la lettre et le sens, rapport où c'est la lettre qui “absorbe” le sens » (BERMAN, 1999, p. 67).

En contrepartie, la traduction étant associée à la lecture particulière du traducteur, il semble important de souligner son autonomie en ce qui concerne la formulation du texte-cible, comme le signale le traducteur Hœpffner dans l'extrait qui suit : "I would like to underline something that tends to be easily forgotten: a translation is written by the translator, not by the author ${ }^{1} \gg(2011, \mathrm{p} .32)$. Avec cette déclaration, Hœpffner élève le traducteur au même niveau que l'écrivain. Car, malgré le fait que son écriture soit dérivée, qu'elle soit attachée à un texte-source

\footnotetext{
${ }^{1}$ Ici, j'aimerais souligner quelque chose qui a tendance à être facilement oublié: une traduction est écrite par le traducteur et non pas par l'auteur. (Notre traduction)
} 
qui lui permet d'exister, Antoine Berman affirme que "le traducteur, "laissé à lui-même", peut aussi faire “ce qu'il veut" »(BERMAN, 1995, p. 47). L'écriture du texte-cible, fruit de sa lecture, lui appartient. Le traducteur devient lui aussi un auteur, même si « c'est un écrivain qui n'a pas à affronter la page blanche » (HCEPFFNER, 2011, Op. cit. p.32).

Cependant, le statut du traducteur n'est pas si facilement défini, comme l'on peut le constater à travers la constellation d'œuvres théoriques qui mettent le travail du traducteur au centre de la discussion. Déployée sous plusieurs formules qui vont de la Lettre et l'Esprit, en passant par les «verres transparents » et les "verres colorés » de Georges Mounin, «l'équivalence dynamique » et «l'équivalence formelle » d'Eugène A. Nida, ou encore par les « ciblistes » et les « sourciers » de Jean-René Ladmiral, la tâche du traducteur ne cesse d'être remise en question. Malgré les multiples définitions données par ces grands théoriciens cités plus haut, la nature de ces couples conceptuels tourne autour d'une question commune qui touche, entre autres, à l'approche du traducteur envers le texte source, sa lecture, son interprétation et surtout ses choix traductifs, car en ayant à sa disposition deux chemins opposés et selon Ladmiral incompatibles devant lui (2006, p.133), le traducteur doit choisir entre produire un texte où la langue-source occupe une place importante en dépit de la fluidité du texte en langue cible, ou bien adapter le texte-source à la langue cible afin d'accorder une naturalité à l'œuvre traduite.

Selon Umberto Eco, ce choix décisif est, en grande partie, dû à la lecture que le traducteur fait du texte-source, à partir de laquelle il décidera quel aspect de l'œuvre il faudra mettre en évidence en dépit d'un autre, car en traduction il faut choisir, comme le suggère aussi Ladmiral (1987, p. 190-197). Eco développe cette idée dans son célèbre Dire presque la même chose (2003) comme suit :

Une traduction oriente toujours à un certain type de lecture de l'œuvre, comme le fait la critique proprement dite, parce que, si le traducteur a négocié en choisissant de porter son attention sur certains niveaux de lecture du texte, il a automatiquement focalisé sur eux l'attention du lecteur. Dans ce sens les traductions de la même œuvre s'intègrent entre elles, car souvent elles nous amènent à voir l'original sous points de vue différents (ECO, 2010, p. 312)

À cet égard, le premier mot du roman est, particulièrement, pertinent à la discussion. Inutile de souligner l'importance de l'incipit d'une œuvre, cette phrase étant le premier contact du lecteur avec ce nouveau monde littéraire qui s'ouvre devant lui. Par surcroît, c'est le moment où s'établit le pacte de lecture. Or, dans le cas de Grande Sertão : Veredas, notre regard tombe sur le mot «nonada », qui, à première vue, ne dit pas grand-chose, ce mot n'étant pas familier de la 
Traduction ou la lecture en profondeur : analyse des traductions françaises de Grande Sertão : Veredas de João Guimarães Rosa

plupart des lecteurs brésiliens, le regard du lecteur demeure, donc, flou vis-à-vis du texte. Cependant, en poursuivant la lecture de l'œuvre, l'on s'habitue, au fur et à mesure, à cette forme d'écriture, pourtant si éloignée de la langue standard. L'on n'a pas pour autant accès à toute sa dimension linguistique, comme l'explique Marcelo Marinho en affirmant qu'il s'agit d'une intention déclarée de l'auteur:

Les mots sont choisis par Guimarães Rosa de façon à laisser le texte vague et imprécis, de façon à conduire le lecteur au beau milieu d'une forêt envoutante (la dantesque selva oscura) de signes sonores dont le sens (dénotatif) précis n'a pas beaucoup d'importance à la première lecture. (2003, p. 94)

De ce riche extrait, il faut retenir la remarque que Marinho fait sur la première lecture, car si initialement ce mot, " nonada », est avalé par les innovations qui se suivent à chaque page, une lecture attentive, telle que celle du traducteur, permettra de remarquer sa reprise constante. Or, seulement une lecture naïve ignorerait la pertinence de cette reprise, qui, d'ailleurs, ouvre et clôt le roman. Cependant, l'on voit dans les deux traductions une inattention à la récurrence de ce mot qui apparaît à des moments décisifs du récit, et qui est traduit arbitrairement, comme l'on remarque dans le tableau qui suit :

\begin{tabular}{|l|l|l|}
\hline JGR, 1956 & JJV, 1965 & MLP, 1996 \\
\hline Nonada. (p. 9) & Foutaises ! (p. 11) & Que nenni ! (p. 21) \\
\hline Nonada ! (p. 311) & Foutaises ! (p. 225) & Que nenni (p. 265) \\
\hline Nonada. (p. 326) & Bêtises (p. 237) & Que nenni. (p. 277) \\
\hline Nonada. (p. 411) & Des foutaises (p. 397) & Des sornettes (p. 456) \\
\hline Nonada. (p. 595) & Quelque peu (p. 430) & Broutilles (p. 487) \\
\hline Nonada. (p. 608) & Foutaises (p. 439) & Que nenni (p. 497) \\
\hline
\end{tabular}

Source : l'auteure

De fait, João Guimarães Rosa utilise le même terme dans des contextes divers, il attribue des nouvelles connotations à des mots inattendus, il trouble le lecteur en reformulant son lexique, en transformant des mots qui figuraient déjà différemment dans le roman, ou comme Marinho l'observe sensiblement :

Le romancier s'abstient de convaincre le lecteur, car il le souhaite plutôt complice, compagnon de perplexité devant l'univers. Il ne propose pas de solutions ou de résolutions, mais plutôt des prises de possession par l'intermédiaire de la création, geste arbitraire qui procure de l'ambiguiité, qui recrée un univers fragmentaire qui se veut un et indivisible. (P. 102) 
Ce nouvel emploi du même mot se vérifie notamment dans l'avantdernière apparition de «nonada », où les deux traducteurs sortent de leur cadre traductif habituel, où «nonada », qui est communément traduit par « foutaises » et «que nenni », respectivement, devient «quelque peu » et «broutilles ». Ce changement lexical dans les traductions provient d'une envie de clarté de la part des traducteurs, d'adaptation du vocable à ce contexte précis. En l'occurrence Guimarães Rosa écrit «O senhor nonada conhece de mim », traduit par Villard comme suit "Monsieur en connaît quelque peu sur moi », et par LapougePettorelli «Vous ne connaissez que broutilles de moi ».

En effet, Grande Sertão: Veredas est surchargé d'expressions qui réapparaissent constamment pendant tout le récit et qui deviennent une sorte d'identifiant linguistique de Riobaldo, tel un tatouage. L'une des plus évidentes dans le roman est, sans doute, "mire veja » réitérée quarante-cinq fois, comme une sorte d'incantation qui, dès qu'elle est prononcée, avertit et prépare le lecteur aux aventures du Narrateur. Ce n'est jamais « pour rien » qu'il la prononce, elle est toujours suivie soit $d^{\prime}$ un épisode fantastique de sa vie, $d^{\prime}$ une leçon morale ou $d^{\prime}$ un avertissement : il faut s'y attendre. Cependant, cette fonction d'introduction qu'elle assume dans le récit trouve ses origines dans la répétition, qui la consolide et la met en évidence. Or, ne pas reproduire cette spécificité de l'œuvre en languecible, c'est effacer une portion importante de ce qui constitue sa richesse linguistique.

Plutôt que de transcrire toutes les variations de cette expression rencontrées dans les traductions, ce qui serait long et sans grand intérêt, le but n'étant pas de produire un travail de catalogage de différences, mais de réfléchir sur la traduction de certains aspects récurrents à l'œuvre; l'on a réduit à sept le nombre de variations exposées, afin d'illustrer notre propos sans pour autant se perdre dans les exemples :

\begin{tabular}{|l|l|l|}
\hline JGR, 1956 & JJV, 1965 & MLP, 1996 \\
\hline Mire veja (p. 15) & Tenez (p. 15) & Voyez plutôt (p. 28) \\
\hline Mire veja (p. 25) & X (p. 22) & Ecoutez voir (p. 36) \\
\hline Mire veja (p. 41) & Voyez donc (p. 33) & Ecoutez voir (p. 49) \\
\hline Mire veja (p. 61) & Tenez (p. 48) & Ecoutez ça (p. 66) \\
\hline Mire veja (p. 65) & Voyez (p. 51) & Voyez plutôt (p. 70) \\
\hline $\begin{array}{l}\text { Mire veja, mire veja (p. } \\
\text { 364) }\end{array}$ & Voyez donc ça (p. 263) & $\begin{array}{l}\text { Voyez bien, voyez bien } \\
\text { (p. 306) }\end{array}$ \\
\hline Mire veja (p. 607) & Voyez-vous (p. 439) & Ecoutez voir (p. 497) \\
\hline
\end{tabular}

Source : 1 'auteure

Ce sujet me fait penser au titre de l'ouvrage d'Umberto Eco, Dire presque la même chose, car ces exemples nous prouvent qu'il s'agit réellement de dire presque la même chose. Toutefois, si ce presque arrive à garder le sens de 
l'expression, il viole « la règle de ne jamais enrichir le lexique de l'auteur, même quand cela est tentant » (ECO, 2003, p. 119). En parlant de sa propre expérience de traducteur de Sylvie, de Nerval, Umberto Eco observe que le langage de Sylvie est « lexicalement pauvre », ce qui oblige parfois le traducteur à le varier, lorsque cette pauvreté lexicale instaure une ambiguïté dans le texte-cible. Or, dans les exemples qui nous concernent, l'on constate qu'il s'agit moins d'une nécessité, au nom de l'intelligibilité du texte, que d'une envie « d'enrichir » le texte-source, $c^{\prime}$ est la tentation même dont parle Eco.

Il semble donc qu'après tout, la traduction soit une question de point de vue, car une version ne peut enfermer toutes les lectures possibles d'une œuvre, et les retraductions en sont la preuve. Elles témoignent des multiples façons de traduire en renforçant l'idée proustienne selon laquelle il y a autant de versions d'un livre qu'il y a de lecteurs. À cet égard, l'exemple qui suit peut être relevant :

\begin{tabular}{|c|c|c|}
\hline$R, 1956$, p. 594 & JJV, 1956, p. 439 & MLP, 1996, p. 497 \\
\hline $\begin{array}{l}\text { Amável o senhor me } \\
\text { ouviu, minha idéia } \\
\text { confirmou: que o Diabo } \\
\text { não existe. Pois não? O } \\
\text { senhor é um homem } \\
\text { soberano, circunspecto. } \\
\text { Amigos somos. } \\
\text { Nonada. O diabo não } \\
\text { há! É o que eu digo, se } \\
\text { for.. Existe é homem } \\
\text { humano. Travessia. }\end{array}$ & $\begin{array}{l}\text { Monsieur a été bien } \\
\text { aimable } \\
\text { de m'écouter, il a } \\
\text { confirmé mon idée que } \\
\text { le Diable existe pas. } \\
\text { S'pas? Monsieur est un } \\
\text { homme de qualité, } \\
\text { avisé. Nous sommes } \\
\text { amis. Foutaises. Y a pas } \\
\text { de diable! C'est ce que } \\
\text { je dis, à moins qu'il } \\
\text { existerait... } \\
\text { qu'existe, Ce cest } \\
\text { l'homme, l'être humain. } \\
\text { Un passage. }\end{array}$ & $\begin{array}{l}\text { Vous m'avez écouté } \\
\text { bien aimable, vous avez } \\
\text { confirmé mon idée : que } \\
\text { le Diable n'existe pas. } \\
\text { N'est-ce pas? Vous êtes } \\
\text { un homme souverain, } \\
\text { circonspect. Nous } \\
\text { sommes amis. Que } \\
\text { nenni ! Le diable } \\
\text { n'existe pas! C'est ce } \\
\text { que je dis, quand bien } \\
\text { même... Ce qui existe, } \\
\text { c'est l'homme humain. } \\
\text { Traversée. }\end{array}$ \\
\hline
\end{tabular}

Source : l'auteure

Je dirais que cette phrase résume toutes les problématiques du roman au niveau linguistique, de la syntaxe bouleversante à la néologie, en passant par le rythme et l'oralité, la présence des mots-clés qui constituent les systématismes, tout cela forme un concentré de langue non standard qui clôt Grande Sertão: Veredas. Tout d'abord, l'on revient à la question syntaxique de l'écriture rosienne, où encore une fois « le romancier ne fait qu' intensifier un processus de déviation face aux normes savantes de la langue» (MARNHO, 2003, p. 95). Cependant, les deux traducteurs réécrivent le texte-cible sous le primat normatif, en clarifiant la construction imbriquée de l'original. Cette tendance à procéder à une réorganisation de la phrase en langue-source, et à traduire à partir de ce texte (pré)modifié, ce que Jakobson appelle la traduction intralinguale, ou 
reformulation, oriente le lecteur-cible vers d'autres aspects de l'oeuvre. En ce sens, chaque traduction propose une nouvelle lecture du même texte-source, dorénavant redirigé vers d'autres thèmes. Cela peut être constaté dans les traductions françaises de Grande Sertão : Veredas, où chaque traducteur adopte sa propre manière de traduire, en privilégiant de différents aspects de l'oeuvre, ce qui crée deux textes légitimes et complémentaires, en rejoignant l'idée d'Umberto Eco présentée plus haut.

Ainsi, une personne qui a eu l'opportunité de lire les deux traductions françaises de Grande Sertão: Veredas aura deux points de vue différents, voire opposés, sur la même œuvre, qu'elle ne connait pas directement, et qui permettra à son tour, si un jour ce même lecteur accède au texte-source, d'en faire une troisième lecture.

Avec la première traduction, qui remplace la langue de Riobaldo par un parler populaire paysan, inspiré de sa région normande, Jean-Jacques Villard met en place une écriture chargée de traits propres à la langue parlée, élaborée surtout à travers une sorte de transcription phonétique, à la manière de Raymond Queneau, sans pour autant produire des déviations du même niveau d'audace. En effet, il se limite à supprimer la double négation, ou plus rarement, à créer une forme déviante à partir d'un mot, ou d'une expression, standard du texte-source, tel est le cas de « $S^{\prime}$ pas ? », qui ne trouve pas son équivalent irrégulier en languesource.

Villard poursuit en rationalisant l'écriture rosienne jusqu'au dernier mot du roman, comme on peut le voir dans l'extrait en question. Les redondances, telle " homem humano », les inversions syntaxiques, et autres signes qui nous permettent de reconnaître le texte-source de par ses spécificités sont pour autant transformées, dans un souci de littérarisation qui, selon Oseki-Dépré, est commun au traducteur français :

Le traducteur français vise à la littérarisation (adéquation entre sa traduction et le système littéraire français), à la transparence du sens, quitte à réorganiser les "constructions enchevêtrées », " tortueuses ", " baroques » fréquemment présentes dans les textes en provenance de la littérature latino-américaine (OSEKI-DEPRE apud BALLARD, 2011, p. 20).

En effet, les discours non standard en langue française n'intéressent que marginalement les théoriciens, et s'affirment en tant que véritable défi pour les traducteurs habitués à un modèle normatif d'écriture, comme le signale Karen Bruneaud-Wheal : 
Dans la traduction de sociolectes littéraires, c'est-à-dire de faits de langues dits «nonstandard », le traducteur subit plus que jamais la pression de ce paradoxe qui lui demande de choisir entre le possible, qui consisterait à étirer, modeler, faire violence à la langue de réception pour lui faire restituer l'étrangeté de l'autre, et celle lui demandant d'être lisible, acceptable - et vendable - avant tout (2011, p. 49).

L'extrait tiré de l'article de Bruneaud-Wheal sur la traduction de sociolectes littéraires touche à une question primordiale : le degré d'acceptation du lecteur français de cette écriture-déviance, ainsi que l'intérêt commercial de vente d'un produit marginal par rapport au modèle d'écriture en littérature française. Autrement dit, il ne suffit pas de bouleverser la langue française et de « lui infliger d'impossibles contorsions pour lui faire dire ce qu'elle ne dirait jamais spontanément au départ » (WUILMART, 2006, p. 146); mais de créer une troisième langue, idée défendue d'ailleurs par plusieurs théoriciens et écrivains, tels que Wuilmart, Casanova, Queneau, ainsi que João Guimarães Rosa. En ce qui concerne aux traductions françaises de Grande Sertão : Veredas, il semble que cette langue serait le résultat de l'interaction des deux langues concernées, de la contribution réciproque du portugais de Rosa, de Mário de Andrade, de Lispector, de l'homme simple, du sertanejo, et du français de Rabelais, ainsi que celui de Queneau, de Proust, du paysan, mais aussi du pidgin, du créole, etc. Rien que le contact de ces formes linguistiques complexes et le recours à différents registres à l'intérieur d'une même langue, permettent de fournir la matière nécessaire pour reproduire l'écriture non standard de JGR en traduction. À cet égard, l'on pourrait paraphraser l'affirmation de Ladmiral : " on ne traduit pas de l'allemand, mais Goethe », et faire remarquer que l'on ne traduit pas du portugais - non plus - mais João Guimarães Rosa.

\section{Considérations finales}

La lingua dovrebbe essere trattata come une concezione del mondo, come l'espressione di una concezione del mondo ${ }^{2}$.

Antonio Gramsci

Les multiples retraductions d'une même œuvre, ainsi que les diverses études critiques de traductions des grands auteurs, comme le cas de Proust, Shakespeare, Baudelaire, Freud et tant d'autres, dont les œuvres sont, encore aujourd'hui, l'objet d'étude de plusieurs œuvres théoriques consacrées à la

2 GRAMSCI, Antonio. Quaderni del Carcere. In: GOBARD, Henri. L'aliénation linguistique. Paris, Flammarion, 1976. «La langue devrait être traitée comme une conception du monde, comme l'expression d'une conception du monde ». 
traduction, confirment le caractère hétérogène de la traduction. En opérant une analyse contrastive entre le texte-source et le(s) texte(s)-cible, ces théoriciens cherchent à retracer les pas du traducteur à la recherche de réponses aux questions posées plus haut dans ce travail et d'autres encore, afin de comprendre le processus traductif propre à chacun des traducteurs pour arriver au résultat final.

Pour certains théoriciens, cités dans ce travail, la tâche du traducteur peut être associée à celle du lecteur comme l'affirme, notamment, Plassard lorsqu'il dit que «Le travail du lecteur, a fortiori du traducteur, c'est le geste mental qui tisse la tresse du texte. Lire, traduire, c'est lier pour reconstruire le texte, le réélaborer, c' est retisser la textualité »(PLASSARD, 2007, p. 265).

Or, en lisant telle phrase selon le concept développé par Proust dans la préface à sa traduction de Sésame et les lys, de Ruskin, intitulée Sur la lecture, lorsqu'il expose l'idée de sa grand-mère en ce qui concerne les textes littéraires, pour qui « le goût d'un seul ne peut pas fixer la vérité » $(1906$, p.12), il semble que l'on puisse étendre telle idée à la traduction et affirmer qu'une lecture/traduction, plus complète soit-elle, ne peut pas être ultime, définitive, unique, car l'œuvre se renouvelle à chaque nouvelle lecture, en nous permettant d'accéder à son essence à travers plusieurs chemins qui se complètent continuellement.

\section{BIBLIOGRAPHIE}

BALLARD, Michel. Censure et traduction. Arras: Artois Presses Université, 2011.

BENJAMIN, Walter. La tâche du traducteur. Traduit de l'allemand par Cédric Cohen Skalli. Paris: Payot \& Rivages, 2011.

BERMAN, Antoine. Jacques Amyot, traducteur français. Essai sur les origines de la traduction en France. Paris: Belin, 2012.

BERMAN, Antoine. La traduction et la lettre ou l'auberge du lointain. (1985) Paris: Seuil, 1999, [1985].

BERMAN, Antoine. Pour une critique des traductions: John Donne. Paris: Gallimard, 1995.

BRUNEAUD-WHEAL, Karen. La double contrainte du traducteur de sociolectes littéraires: entre adéquation et acceptabilité. In: Le double en traduction ou $\mathbf{l}^{\prime}$ 
Traduction ou la lecture en profondeur : analyse des traductions françaises de Grande Sertão :

Veredas de João Guimarães Rosa

(impossible ?) entre-deux. Coll. Dirigée par Michaël Mariaule et Corinne Wecksteen. Arras: Artois Presses Université, 2011.

ECO, Umberto. Dire presque la même chose, expériences de traduction. Traduit par Myriem Bouzahier. Paris: Grasset, 2010 [2003].

GOBARD, Henri. L'aliénation linguistique. Paris: Flammarion, 1976.

HCEPFFNER, Bernard. In: WECKSTEEN, Corinne. De Janus à Ménechme: Portrait du traducteur en agent double. Le double en traduction ou 1'(impossible ?) entre-deux. Artois Presses Université, 2011. p. 32.

LADMIRAL, Jean-René. Traduire: théorèmes pour la traduction. Paris: Gallimard, 2010, [1994].

LADMIRAL, Jean-René. Esquisses conceptuelles, encore... . In: Palimpsestes: traduire ou vouloir garder un peu de la poussière d'or. Paris: Presses Sorbonne Nouvelle, 2006.

LADMIRAL Jean-René, Technique et esthétique de la traduction. Quelle théorie pour la pratique traduisante?, Actes des Journées européennes de la traduction professionnelle, Encrages, n¹7, Paris: Hachette, p. 190-197, 1987.

MARINHO, Marcelo. João Guimarães Rosa. Paris: L'Harmattan, 2003.

PLASSARD, Freddie. Lire pour traduire. Paris: Presses Sorbonne Nouvelle 2007.

PROUST, Marcel. Préface. Sur la lecture. In: RUSKIN, John. Sésame et les lys. Traduit par Marcel Proust. Paris: Mercure de France, 1906 [1865].

ROSA, João Guimarães. Grande Sertão: Veredas. Rio de Janeiro: Livraria José Olympio, 1956.

ROSA, João Guimarães. Diadorim. Traduit du brésilien par Jean-Jacques Villard. Paris: Albin Michel, 1965.

ROSA, João Guimarães. Diadorim. Traduit du brésilien par Maryvonne Lapouge-Pettorelli. Paris: Albin Michel, 1991.

WUILMART, François. La traduction littéraire: source d'enrichissement de la langue d'accueil, RiLUnE, n 4, 2006. 
WUILMART, Françoise. Traduire, c'est lire. TransLittérature, Hiver, n² 20, 2000.

Data de envio: 04/06/2020

Data de aprovação: 21/07/2020

Data de publicação: 21/12/2020 